\title{
Dieta del Caracara Crestado Norteño Caracara cheriway (Falconiformes: Falconidae) en el archipiélago de Jambelí, suroeste de Ecuador
}

\author{
Adrian Orihuela-Torres ${ }^{1 *}$, Leonardo Ordóñez-Delgado ${ }^{1}$, Jorge Brito ${ }^{2,3}$ \\ ${ }^{1}$ Laboratorio de Ecología Tropical y Servicios Ecosistémicos - EcoSs Lab, Departamento de Ciencias Biológicas, \\ Universidad Técnica Particular de Loja, Loja, Ecuador. \\ ${ }_{2}^{2}$ Departamento de Biología, Facultad de Ciencias, Escuela Politécnica Nacional, Quito, Ecuador. \\ ${ }^{3}$ Instituto Nacional de Biodiversidad, Quito, Ecuador. \\ *Autor para correspondencia: adrian.orihuela89@gmail.com \\ Editado por/Edited by: Diego F. Cisneros-Heredia \\ Recibido/Received: 12 Septiembre 2018 Aceptado/Accepted: 9 Noviembre 2018 \\ Publicado en línea/Published online: 22 Noviembre 2019
}

Diet of Northern Crested Caracara Caracara cheriway (Falconiformes: Falconidae) in the Jambelí Archipelago, southwestern Ecuador

\section{Resumen}

Presentamos el primer reporte sobre la ecología trófica del Caracara Crestado Norteño Caracara cheriway en Ecuador, por medio del análisis de egagrópilas colectadas en el archipiélago de Jambelí, provincia de El Oro. Las presas más representativas fueron los mamíferos $(39,4 \%)$, seguidos de insectos $(29 \%)$, reptiles $(13,2 \%)$ y aves $(10,5 \%)$. Los mamíferos aportaron el mayor porcentaje de biomasa $(83,3 \%)$, seguidos por reptiles $(8,5 \%)$ y aves $(7,3 \%)$. Si bien $C$. cheriway es un consumidor generalista altamente oportunista, su dieta se constituyó principalmente de presas vivas en nuestra área de estudio, quedando en segundo plano el consumo de carroña.

Palabras clave: Caracara cheriway, ecología trófica, Ecuador, egagrópilas, Falconidae, Jambelí.

\begin{abstract}
We present the first report of the trophic ecology of Northern Crested Caracara Caracara cheriway in Ecuador, from the analysis of pellets collected at Jambelí archipelago, province of El Oro. The most representative prey were mammals $(39.4 \%)$, followed by insects (29\%), reptiles (13.2\%) and birds (10.5\%). Mammals contributed the highest percentage of biomass $(83.3 \%)$, followed by reptiles $(8.5 \%)$ and birds $(7.3 \%)$. Although $C$. cheriway is a highly opportunistic generalist consumer, its diet was mainly constituted by living prey in our study area, leaving carrion consumption as a complementary feeding strategy.
\end{abstract}

Keywords: Caracara cheriway, Ecuador, Falconidae, Jambelí, pellets, trophic ecology.

\section{INTRODUCCIÓN}

Los estudios de ecología trófica son fundamentales para dilucidar patrones en el funcionamiento de un ecosistema. Estas investigaciones permiten, por ejemplo, establecer cómo se regulan las poblaciones animales en una localidad, reconocer las estrategias de comportamiento alimenticio y, en el caso de depredadores, determinar su influencia en la estructura de la cadena alimenticia (Bó et al., 2007).

Los caracaras (Falconidae) son especies omnívoras con un amplio espectro de elementos de los cuales se alimentan (White et al., 2017). Dentro de este grupo, el Caracara Crestado Norteño Caracara cheriway no es la excepción. Se considera un ave de alimentación generalista, altamente oportunista, que consume una gran variedad de vertebrados vivos, invertebrados y carroña (del Hoyo et al., 2017). Caracara cheriway tiene amplia distribución geográfica, desde Florida y el suroeste de Estados Unidos hasta el noroeste de Perú y la parte media y baja del río Amazonas, en el norte de Brasil (BirdLife International, 2016). En Ecuador, es una especie poco 
común a común en el suroeste; hacia el norte alcanza el sur de la provincia de Los Ríos, y existen unos pocos registros en el valle central de los Andes a 2000 y hasta 3000 m (Ridgely \& Greenfield, 2001).

La dieta de $C$. cheriway ha sido estudiada principalmente en Norteamérica (Rodríguez-Estrella \& RiveraRodríguez, 1997; Morrison et al., 2008; Skoruppa \& Lee, 2008; Morrison \& Dwyer, 2012), y existen también algunas observaciones puntuales en México (Partida \& Rodríguez-Estrella, 2015; Pérez-Estrada \& RodríguezEstrella, 2016; García-Mata et al., 2017). A pesar de ser una especie común en Ecuador, su ecología trófica en el país es desconocida y, hasta donde conocemos, también en el resto de Sudamérica. El presente trabajo se fundamenta en el análisis de egagrópilas colectadas en el archipiélago de Jambelí, provincia de El Oro, suroeste de Ecuador, y se orienta a incrementar el conocimiento sobre el uso de recursos por parte de $C$. cheriway en esta zona insular, tema fundamental para comprender las estrategias de alimentación y dinámica de diferentes especies en los ecosistemas (Pozo-Zamora et al., 2017).

\section{MÉTODOS}

Recolectamos egagrópilas de Caracara cheriway en septiembre de 2016, fuera de la época de reproducción, bajo un nido (Fig. 1a) localizado en el archipiélago de Jambelí, provincia de El Oro, suroeste de Ecuador (3,378480, -80,130430; 5 m s.n.m.). El nido estaba en la intersección de las ramas de un cactus Armatocereus cartwrightianus, a 5,5 m sobre el suelo (Fig. 1b). Estaba construido exclusivamente con ramas secas de 1-2 cm de diámetro, con una forma no muy definida, como es típico de esta especie (Ferguson-Lees \& Christie, 2001). A pesar de que el nido no estaba activo, se podía ver a la pareja de adultos en los alrededores o incluso posados en el cactus, y es posible que lo estuvieran preparando para la próxima época de reproducción (Fig. 1b). El hábitat donde se encontraba el nido corresponde a un arbustal deciduo en zonas adyacentes a playas de arena o playas rocosas (Cerón, 2013). La vegetación era achaparrada, de 2-4 m de altura, y estaba compuesta por plantas herbáceas, rastreras o trepadoras como Cryptocarpus pyriformis, Hippomane mancinella, Scutia spicata, Vallesia glabra, Batis maritima, Canavalia maritima, Ipomoea pescaprae y Sesuvium portulacastrum (Cerón, 2013).

El análisis de las egagrópilas se realizó en el laboratorio, donde medimos la longitud y ancho de cada egagrópila utilizando un calibrador digital (precisión $0,01 \mathrm{~mm}$ ). El peso en seco se midió con una balanza de precisión (Sartorius LA-230P). Disgregamos las egagrópilas según la propuesta de Marti et al. (2007), y colocamos los contenidos en placas Petri para su identificación y cuantificación. El Número Mínimo de Individuos (NMI) fue determinado por el conteo de mandíbulas homólogas o restos de cráneos para los vertebrados; mientras que para artrópodos se usó élitros, cabezas y mandíbulas (no otras partes para evitar reconteo; Manning \& Jones, 1990). La composición de dieta se expresó como frecuencia relativa (NMI de cada tipo de presa dividido por el número total de presas y multiplicado por 100; Grayson 1984, Formoso et al., 2012). La masa promedio (en gramos) de las presas se obtuvo de fuentes secundarias: Brito et al. (2018) para los mamíferos y R. Vargas et al. (datos no publ.) para los reptiles, insectos y arácnidos. Para el cálculo de la biomasa se multiplicó la masa promedio (en gramos) de las especies consumidas por el Número Mínimo de Individuos (NMI) de la especie (Herrera \& Jaksic, 1980). Los componentes alimenticios fueron identificados mediante el uso de guías disponibles y aplicables para la zona de estudio (Brito et al., 2018; Torres-Carvajal et al., 2018) y por comparaciones con material de referencia depositado en el Museo de la Escuela Politécnica Nacional (MEPN) y en el museo del Instituto Nacional de Biodiversidad (MECN).

\section{RESULTADOS}

Colectamos y analizamos un total de 17 egagrópilas con las siguientes dimensiones: largo 3,96 0 0,59 cm (3,34$5,05 \mathrm{~cm})$, ancho $2,42 \pm 0,23 \mathrm{~cm}(2,06-2,7 \mathrm{~cm})$, peso 4,02 $\pm 1,34 \mathrm{~g}(2-5,57 \mathrm{~g})$. Contabilizamos 38 presas de al menos 10 especies diferentes, pertenecientes a cinco clases taxonómicas: mamíferos, aves, reptiles, insectos y arácnidos. El tamaño de las presas varió desde Rattus rattus (160 g) hasta pequeños grillos y escorpiones de menos de un gramo.

Los mamíferos fueron las presas más abundantes en la dieta de $C$. cheriway en nuestro estudio (39,4\% NMI; $83,3 \%$ biomasa). El resto de la dieta la complementan reptiles y aves, con 8,5\% y 7,3\% en aporte de biomasa, respectivamente (Tabla 1). La presa de mamífero más consumida fue Mus musculus. Las especies de roedores Rattus rattus y Aegialomys xanthaeolus fueron menos consumidas, pero aportaron mayor biomasa debido a su mayor tamaño (Tabla 1). Otra especie importante en la dieta fue el lagarto Dicrodon guttulatum, que aportó 
$6,1 \%$ de la biomasa total. Aunque los invertebrados (insectos y arácnidos) aportaron un porcentaje significativo en número de individuos $(36,9 \%)$, tan solo representaron $1 \%$ de la biomasa.

Figura 1: (a) Nido del Caracara Crestado Norteño Caracara cheriway sobre un cactus Armatocereus cartwrightianus en el archipiélago de Jambelí, suroeste de Ecuador (b) Individuos adultos posados sobre el nido (A. Orihuela-Torres).

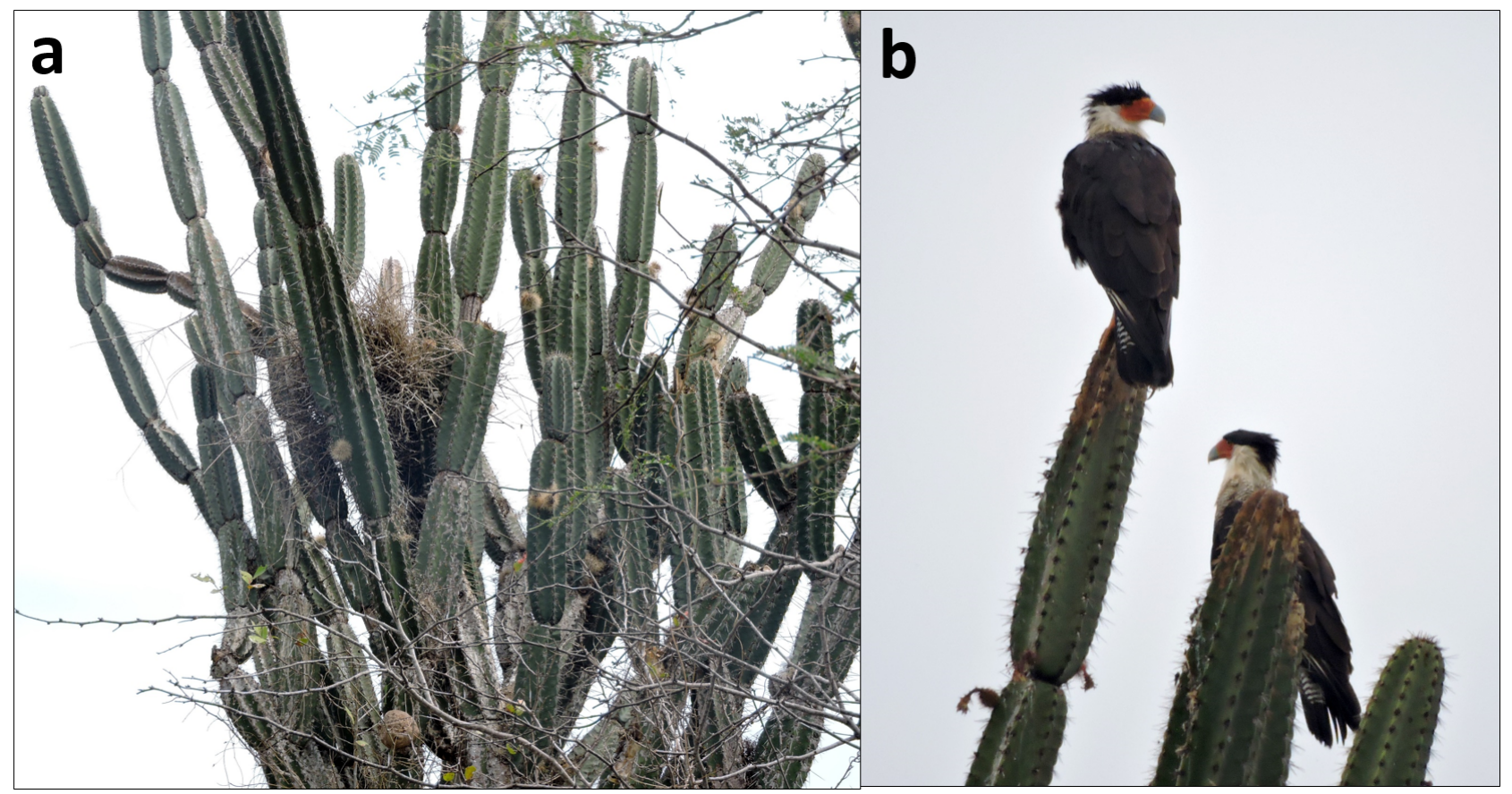

\section{DISCUSIÓN}

Caracara cheriway es un ave carroñera y oportunista, capaz de variar su dieta de manera significativa dependiendo del lugar donde se encuentre y de adaptarse a la disponibilidad de recursos (Morrison \& Pias, 2008). Además de alimentarse de gran variedad de presas vivas, emplea métodos bastante sofisticados para encontrar comida. Se ha reportado que puede cleptoparasitar a rapaces y pelícanos (Rodríguez-Estrella \& Rivera-Rodríguez, 1992; Ferguson-Lees \& Christie, 2001), alimentarse de frutos (Skoruppa \& Lee, 2008) o seguir a automóviles y trenes para alimentarse de las presas que estos atropellan (Ferguson-Lees \& Christie, 2001).

En nuestro estudio, los vertebrados fueron las presas más importantes para $C$. cheriway en un ambiente insular. Estos resultados son similares a otros estudios en los cuales los vertebrados, en especial mamíferos, fueron la parte más importante de su dieta (Rodríguez-Estrella \& Rodríguez, 1997; Morrison \& Pias, 2006; del Hoyo et $a l ., 2017)$. Los resultados de este estudio corroboran la predilección de $C$. cheriway por presas vivas (Morrison \& Dwyer, 2012), pese a que puede consumir carroña cuando tiene la oportunidad. Los artrópodos no fueron un componente importante de la dieta de esta especie en nuestra zona de estudio.

Un dato interesante fue el registro de roedores autóctonos en la dieta de $C$. cheriway. Si bien las especies introducidas Mus musculus y Rattus rattus fueron más abundantes, registramos dos especies nativas: Aegialomys xanthaeolus y Phyllotis sp. Esto es relevante ya que no se habían detectado roedores nativos en otros estudios de dietas de rapaces en la misma zona (Orihuela-Torres et al., 2018) y en otras observaciones de campo no publicadas. Recomendamos que se priorice el levantamiento de información sobre la riqueza y diversidad de los mamíferos en la isla para clarificar estrategias adecuadas de conservación en esta zona, que enfrenta una compleja problemática ambiental y socioeconómica, principalmente por la agresiva actividad camaronera. 
Tabla 1. Composición de la dieta del Caracara Crestado Norteño Caracara cheriway en el archipiélago de Jambelí, provincia de El Oro, Ecuador. NMI = Número mínimo de individuos consumidos.

\begin{tabular}{|c|c|c|c|}
\hline Orden/Familia/Especie & $\begin{array}{l}\text { Peso/individuo } \\
\text { (en g) }\end{array}$ & NMI (\%) & Biomasa en $\mathrm{g}(\%)$ \\
\hline \multicolumn{4}{|l|}{ Rodentia } \\
\hline \multicolumn{4}{|l|}{ Cricetidae } \\
\hline Aegialomys xanthaeolus & 70 & $2(5,3)$ & $140(12,8)$ \\
\hline Phyllotis sp. & 15 & $3(7,9)$ & $45(4,1)$ \\
\hline \multicolumn{4}{|l|}{ Muridae } \\
\hline Mus musculus & 14 & $6(15,8)$ & $84(7,7)$ \\
\hline Rattus rattus & 160 & $4(10,5)$ & $640(58,7)$ \\
\hline MAMMALIA & & $15(39,4)$ & $909(83,3)$ \\
\hline \multicolumn{4}{|l|}{ Passeriformes } \\
\hline no identificados & 20 & $4(10,5)$ & $80(7,3)$ \\
\hline AVES & & $4(10,5)$ & $80(7,3)$ \\
\hline \multicolumn{4}{|l|}{ Squamata } \\
\hline \multicolumn{4}{|l|}{ Tropiduridae } \\
\hline Microlophus occipitalis & 13 & $2(5,3)$ & $26(2,4)$ \\
\hline \multicolumn{4}{|l|}{ Teiidae } \\
\hline Dicrodon guttulatum & 22 & $3(7,9)$ & $66(6,1)$ \\
\hline REPTILIA & & $5(13,2)$ & $92(8,5)$ \\
\hline \multicolumn{4}{|l|}{ Coleoptera } \\
\hline Carabidae & 1 & $5(13,2)$ & $5(0,5)$ \\
\hline \multicolumn{4}{|l|}{ Orthoptera } \\
\hline Gryllidae & 0,5 & $6(15,8)$ & $3(0,3)$ \\
\hline INSECTA & & $11(29)$ & $8(0,8)$ \\
\hline \multicolumn{4}{|l|}{ Scorpionida } \\
\hline no identificado & 0,5 & $3(7,9)$ & $1,7(0,2)$ \\
\hline ARACHNIDA & & $3(7,9)$ & $1,7(0,2)$ \\
\hline Total & & $38(100)$ & $1090,7(100)$ \\
\hline
\end{tabular}

\section{AGRADECIMIENTOS}

A la Universidad Técnica Particular de Loja por financiar el trabajo de campo en el archipiélago de Jambelí y a Daniel Rosado por revisar el manuscrito, en especial las partes traducidas al inglés. 


\section{REFERENCIAS}

Bó, M. S., Baladrón, A. V., \& Biondi, L. M. (2007). Ecología trófica de Falconiformes y Strigiformes: tiempo de síntesis. El Hornero, 22(2), 97-115. URL: http://ref.scielo.org/qp5cdf

BirdLife International. (2016). Species factsheet: Caracara cheriway. Cambridge, UK: BirdLife International, IUCN Red List for birds. URL: http://datazone.birdlife.org/species/factsheet/crested-caracara-caracaracheriway

Brito, J., Camacho, M.A., Romero, V., \& Vallejo, A. (2018). Mamíferos del Ecuador. Version 2018.0. Museo de Zoología, Pontificia Universidad Católica del Ecuador. URL: https://www.bioweb.bio/faunaweb/mammaliaweb/

Cerón, C. (2013). Arbustal deciduo y Herbazal de playas del Litoral. En: Ministerio del Ambiente del Ecuador. Sistema de clasificación de los ecosistemas del Ecuador continental. (pp. 34-36). Quito: Ministerio del Ambiente del Ecuador.

del Hoyo, J., Collar, N., \& Marks., J.S. (2017). Crested Caracara (Caracara cheriway). En: del Hoyo, J., Elliott, A., Sargatal, J., Christie, D.A. \& de Juana, E. (Eds.). Handbook of the birds of the world alive. Barcelona: Lynx Edicions. URL: http://www.hbw.com/node/467494

Fergusson-Lees, J., \& Christie, D.A. (2001). Raptors of the world. Boston, MS: Houghton Mifflin Company.

Formoso, A.E., Teta, P., \& Cheli, G. 2012. Food habits of the Magellanic Horned Owl (Bubo virginianus magellanicus) at southernmost Patagonia, Argentina. Journal of Raptor Research, 46: 401-406. DOI: https://doi.org/10.3356/JRR-12-22.1

García-Mata, E.S., Cruz-Sáenz, D., Carlos-Gómez, J.A., Navarro-Velázquez, B., Lazcano, D., \& Wilson, L.D. (2017). Notes on the herpetofauna of western Mexico 17: Predation on Rhinella horribilis (Linnaeus, 1758) by two species, Leptodeira maculata (Hallowell, 1861) and Caracara cheriway (Jacquin, 1784), in the municipality of Cuauhtémoc, Colima, Mexico. Bulletin of the Chicago Herpetological Society, 52(8), 139-145. URL: http://www.chicagoherp.org/bulletin/52(8).pdf

Grayson, D.K. (1984). Quantitative zooarchaeology: topics in the analysis of archaeological faunas. Orlando, FL: Academics Press.

Herrera, C.M., \& Jaksic, F.M. (1980). Feeding ecology of the barn owl in Central Chile and southern Spain: a $\begin{array}{lllll}\text { comparative } \quad \text { study. } & \text { 97k, } & \text { 760-767. } & \text { URL: }\end{array}$ http://www.jstor.org/stable/4085747?seq=1\#page scan tab contents

Manning, R.W., \& Jones, J.K. 1990. Remains of small mammals recovered from Barn Owl pellets from Crosby county, Texas. Texas Journal of Science, 42, 311-312.

Marti, C.D., Bechard, M., \& Jaksic, F.M. (2007). Food habits. En D.M. Bird \& K.L. Bildstein (Eds), Raptor research and management techniques manual (pp. 129-151). Blaine, WA: Raptor Research Foundation, Hancock House Publishers.

Morrison, J.L., \& Dwyer, J.F. (2012). Crested caracara (Caracara cheriway). En A. Poole (Ed), The birds of North America online. Ithaca: Cornell Lab of Ornithology. URL: http://bna.birds.cornell.edu/bna/species/249

Morrison, J.L., \& Pias, K.E. (2006). Assessing the vertebrate component of the diet of Florida's Crested Caracaras (Caracara cheriway). Florida Scientist, 69(1), 36-43. URL: http://www.jstor.org/stable/24321424

Morrison, J.L., Pias, K.E., Abrams, J., Gottlieb, I.G., Deyrup, M., \& McMillian, M. (2008). Invertebrate diet of breeding and nonbreeding Crested Caracara (Caracara cheriway) in Florida. Journal of Raptor Research, 42(1), 38-47. DOI: https://doi.org/10.3356/JRR-07-47.1 
Orihuela-Torres, A., Ordóñez-Delgado, L., Brito, J., López, F., Mazón, M., \& Freile, J.F. (2018). Ecología trófica del búho terrestre Athene cunicularia punensis (Strigiformes: Strigidae) en el archipiélago de Jambelí, provincia de El Oro, suroeste de Ecuador. Revista Peruana de Biología, 25(2), 123-130. DOI: http://dx.doi.org/10.15381/rpb.v25i2.13376

Partida, A., \& Rodríguez-Estrella, R. (2015). Evidencia de cletoparasitismo del caracara común (Caracara cheriway) sobre el alcaudón verdugo (Lanius ludovicianus). Acta Zoológica Mexicana, 31(2), 306-308. URL: http://www.scielo.org.mx/scielo.php?script=sci_arttext\&pid=S0065-17372015000200019

Pérez-Estrada, C.J., \& Rodríguez-Estrella, R. (2016). Caracara cheriway predation on migratory waterbirds, Egretta thula and Podiceps nigricollis, in southern Baja California Peninsula. Acta Zoológica Mexicana (nueva serie), 32(1), 129-131. URL: http://www.scielo.org.mx/pdf/azm/v32n1/0065-1737-azm-32-01-00129.pdf

Pozo-Zamora, G.M., Aguirre, J., \& Brito, J. (2017). Dieta del cernícalo americano (Falco sparverius Linnaeus, 1758) en dos localidades del valle interandino del norte de Ecuador. Revista Peruana de Biología, 24(2), 145150. DOI: http://dx.doi.org/10.15381/rpb.v24i2.12305

Ridgely, R.S., \& Greenfield, P.J. (2001). The birds of Ecuador. Volume I: Status, distribution and taxonomy. Ithaca, NY: Cornell University Press.

Rodríguez-Estrella, R., \& Rivera-Rodríguez, L. (1992). Kleptoparasitism and other interactions of Crested Caracara in the cape region, Baja California, Mexico. Journal Field Ornithology, 63(2), 177-180. URL: http://www.jstor.org/stable/4513686

Rodríguez-Estrella, R., \& Rivera-Rodríguez, L. (1997). Crested Caracara food habits in the Cape region of Baja California, Mexico. Journal of Raptor Research, 31(3), 228-233. URL: https://sora.unm.edu/sites/default/files/journals/jrr/v031n03/p00228-p00233.pdf

Skoruppa, M.K., \& Lee, M.C. (2008). Crested Caracaras feed on pecans in South-central Texas. Journal of Raptor Research, 42(4), 299-300. DOI: https://doi.org/10.3356/JRR-08-30.1

Torres-Carvajal, O., Pazmiño-Otamendi, G., \& Salazar-Valenzuela, D. (2018). Reptiles del Ecuador. Versión 2018.0. Museo de Zoología QCAZ, Pontificia Universidad Católica del Ecuador. URL: https://www.bioweb.bio/faunaweb/reptiliaweb/

White, C.M., Olsen, P.D., \& Kiff, L.F. (2017). Falcons and caracaras (Falconidae). En del Hoyo, J., Elliott, A., Sargatal, J., Christie, D.A. \& de Juana, E. (Eds), Handbook of the birds of the world alive. Barcelona: Lynx Edicions. URL: http://www.hbw.com/node/52215 on 7 October 2017). 